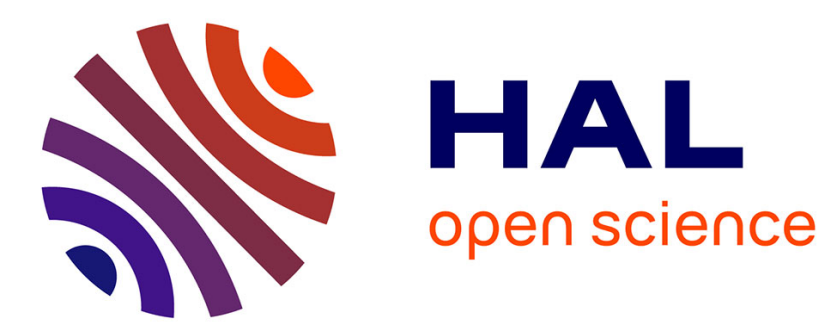

\title{
Online Reconstruction Of Vehicles In A Car Park
}

Christopher Tay, Cédric Pradalier, Christian Laugier

\section{To cite this version:}

Christopher Tay, Cédric Pradalier, Christian Laugier. Online Reconstruction Of Vehicles In A Car Park. Proc. of the Int. Conf. on Field and Service Robotics, Jul 2005, Port Douglas Australia, Australia. pp.207-218, 10.1007/978-3-540-33453-8_18 . inria-00182042

\section{HAL Id: inria-00182042 \\ https://hal.inria.fr/inria-00182042}

Submitted on 24 Oct 2007

HAL is a multi-disciplinary open access archive for the deposit and dissemination of scientific research documents, whether they are published or not. The documents may come from teaching and research institutions in France or abroad, or from public or private research centers.
L'archive ouverte pluridisciplinaire $\mathbf{H A L}$, est destinée au dépôt et à la diffusion de documents scientifiques de niveau recherche, publiés ou non, émanant des établissements d'enseignement et de recherche français ou étrangers, des laboratoires publics ou privés. 


\title{
On-Line Reconstruction of Vehicles In A Car Park
}

\author{
Christopher Tay Meng Keat ${ }^{1}$, Cédric Pradalier ${ }^{2}$, and Christian Laugier ${ }^{3}$ \\ ${ }^{1}$ INRIA Rhône Alpes GRAVIR Laboratory tay@inrialpes.fr \\ 2 CSIRO ICT Center, Canberra-Australia cedric.pradalier@csiro.au \\ 3 INRIA Rhône Alpes GRAVIR Laboratory christian.laugier@inrialpes.fr
}

Summary. In this paper, a method of obtaining vehicle hypothesis based on laser scan data only is proposed. This is implemented on the robotic vehicle, CyCab, for navigation and mapping of the static car park environment. Laser scanner data is used to obtain hypothesis on position and orientation of vehicles with Bayesian Programming. Using the hypothesized vehicle poses as landmarks, CyCab performs Simultaneous Localization And Mapping (SLAM). A final map consisting of the vehicle positions in the car park is obtained.

Key words: Vehicle Detection, Bayesian Programming

\section{Introduction}

In the framework of automatic vehicles in car parks, a 2D map of a car park is constructed using the autonomous vehicle, $\mathrm{CyCab}$, as the experimental platform. The map of the car park will contain the positions and orientations of the different vehicles in the car park. An application of such a map is to serve as a reference to indicate obstacle positions . Furthermore, it can indicate the state of the parking lots in the car park, and possibly be used in higher level applications such as automatic parking.

Several object based representation of the environment were proposed. Chatila et al. [8] represented the map with a set of lines. More advanced methods in mapping consists of approximating the environment using small polygons [3] [4]. Such methods used a variant of the Expectation-Maximization to generate increasingly accurate $3 \mathrm{D}$ maps as more observations are made. In this paper, a higher level of representation (vehicles in this case) of the environment is used instead of fundamental geometrical entities.

Currently, the CyCab robotic vehicle localizes itself in a static environemnt with respect to artificially installed reflective cones. This localization serves to build a grid map of the environment and has the capability to perform motion planning with safe navigation as described in [1]. However, reflective cones as 
artifical landmarks is not a very practical approach. An improvement from an application point of view is to use naturally occuring objects often found in the car park as landmarks. In this paper, vehicles found in the car park are used.

The general idea is to use only the laser data without artificial or predefined landmarks, $\mathrm{CyCab}$ will navigate the car park autonomously while generating a map of its environment.While CyCab is travelling around the car park, scanning the environment, CyCab continuously reads in odometric and laser data. At each stage of the iteration, CyCab estimates its own position and orientation of the form $(x, y, \theta)$ and creates a map of the car park in the world frame of reference. The origin of the world frame of reference is taken from the initial position of CyCab. The map is then represented as a set of tuples, each containing the position and orientation of the vehicles detected. CyCab hypothesizes the configuration of the vehicles in the surrounding based on the laser scan inputs from laser scanner only.

The advantage of the approach is its ability to map any car park without installing any external aids. With the set of vehicle poses representing the map, a compact and semantic representation of the map can be obtained.

\section{System Overview}

The mechanism of the entire system can be broken down into three fundamental components, vehicle detection, the simultaneous localization and mapping(slam) and the map construction. CyCab is provided with two types of raw data, the laser scans and CyCab's odometric data. Figure 1 shows the block diagram of the different stages and its interactions:

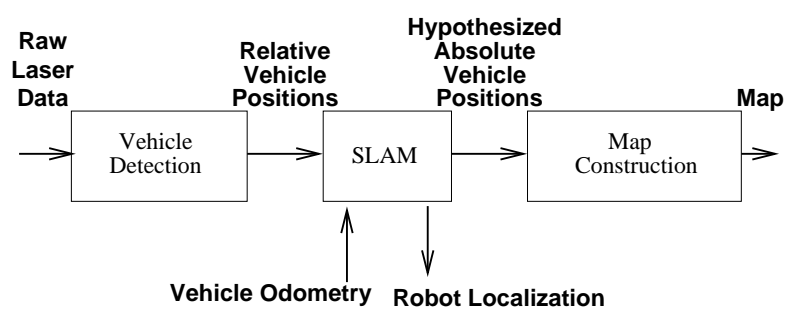

Fig. 1. Overview showing the mapping process

1. Vehicle Detection: With only raw laser scan data, vehicle detection constructs hypotheses about the positions and orientation of vehicles in the car park.

2. SLAM: Coupled with information about odometry of CyCab, SLAM module makes use of the constructed vehicle hypotheses as landmarks 
to localize itself. With its own configuration, SLAM can then calculate the absolute configuration of the vehicles with respect to real world coordinates.

3. Map Construction: The hypotheses of vehicles have to be checked for inconsistencies. It is possible for the hypotheses obtained to conflict with a previous corresponding hypothesis. Furthermore, multiple hypothesis SLAM methods such as FastSLAM produces a set of hypotheses, a map construction module is required to merge the information from the different hypotheses to obtain the final map.

\section{Vehicle Detection}

Vehicle detection is the process of forming possible vehicle hypotheses based on the laser data readings. This problem is addressed in this paper using bayesian programming[2]. Bayesian programs provides us with a framework for encoding our a priori knowledge on the vehicle to infer the possible vehicle poses. In this case, the a priori knowledge consists of the length and width of vehicles which is assumed to be the same across standard vehicles (cars) and that the type of vehicles in the car park is of the same class. The subtle differences in the size of the vehicles can be accomodated for in the bayesian paradigm and this property renders our assumption practical.

The detection of vehicles takes place in two stages. The first stage is basically composed of three portions:

1. Clustering and segmentation, to group a set of points indicating objects, using a distance criterion. Next, segments are obtained using classical split and merge techniques.

2. Vehicle hypotheses construction using bayesian programming. The construction of hypotheses by bayesian programming results in a mechanism similar to that of hough transform. Peak values in the histogram indicates the most probable vehicle poses.

With real data, the first stage produces too many false positives. A second stage of filtering is applied to each vehicle hypothesis obtained after the first stage as a form of validation gating in order to reduce the number of false positives. It is broken down into two portions:

1. Edge Filtering is applied to extract the set of line segments that is only relevant to the vehicle hypothesis in question.

2. Vehicle Support Filtering is based on our proposed metric, vehicle support, that measures how much of the two adjacent sides of a vehicle are seen. We try to remove as many false positives as possible using the vehicle support. 


\subsection{Construction of vehicle hypotheses}

A bayesian program is used to infer vehicle positions. The formulation of our bayesian program results in a mechanism similar to that of a hough transform. We can infer on positions and orientations of vehicles from segments detected from laser scan data which is analogous to the way line segments are recovered from an ensemble of points. However our histogram cells are updated in terms of probability which takes into account the length and the width of vehicles instead of voting in the case of hough transform.

Briefly, a bayesian program is composed of:

- the list of relavant variables;

- a decomposition of the joint distribution over these variables;

- the parametrical form of each factor of the decomposition;

- the identification of the parameters of these parametrical forms;

- a question in the form of probability distribution inferred from the joint distribution.

In the construction of the histogram, each line segment is treated independently. In doing so, it will be sufficient to simply go through the list of segments and add necessary information into the histogram for each line segment. This is achieved by performing data fusion with diagnostic [7]. Inference of vehicle poses is represented by the bayesian program in fig. 2 and fig. 3 . In this paper, the following variables are adopted:

- $V$ : A boolean value indicating the presence of a vehicle

- $Z=(x, y, \theta)$ : The pose of a vehicle

- $\mathbf{S}$ : Ensemble of extracted line segments

- $\mathbf{M} \in\{0,1\}^{p}:$ Compatibility of segments with vehicle pose

- $\mathbf{C} \in\{1,2\}^{p}:$ A value of 1 or 2 if segment corresponds to the width and length of a vehicle respectively

- $\pi$ : A priori knowledge

To represent the absence of specific knowledge on the presence of vehicle $P\left(V \mid \pi_{f}\right)$, the pose of the vehicle $P\left(Z \mid \pi_{f}\right)$ and the segments $P\left(\mathbf{S} \mid \pi_{f}\right)$, they are represented as a uniform distribution.

The semantic of the question from the bayesian program (fig. 2) is to find the probability of a vehicle given all segments and the vehicle pose. This question can be simplified using baye's rule:

$$
\begin{aligned}
& P\left([V=1] \mid[\mathbf{M}=\mathbf{1}][\mathbf{S}] Z \pi_{f}\right) \\
= & K \prod_{i} P\left(\left[M_{i}=1\right] \mid[V=1] Z S_{i} \pi_{f}\right)
\end{aligned}
$$

With K constant. The simplification of the question gives the product of the probability of the contributions of each line segment, which is given by each sensor sub-model. The local maximas of the function, $P([V=1] \mid[\mathbf{M}=\mathbf{1}][\mathbf{S}=$ 


\begin{tabular}{|c|c|c|}
\hline 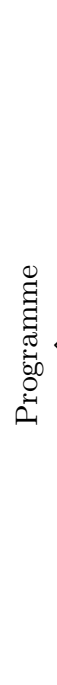 & 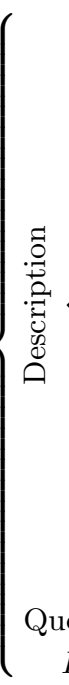 & 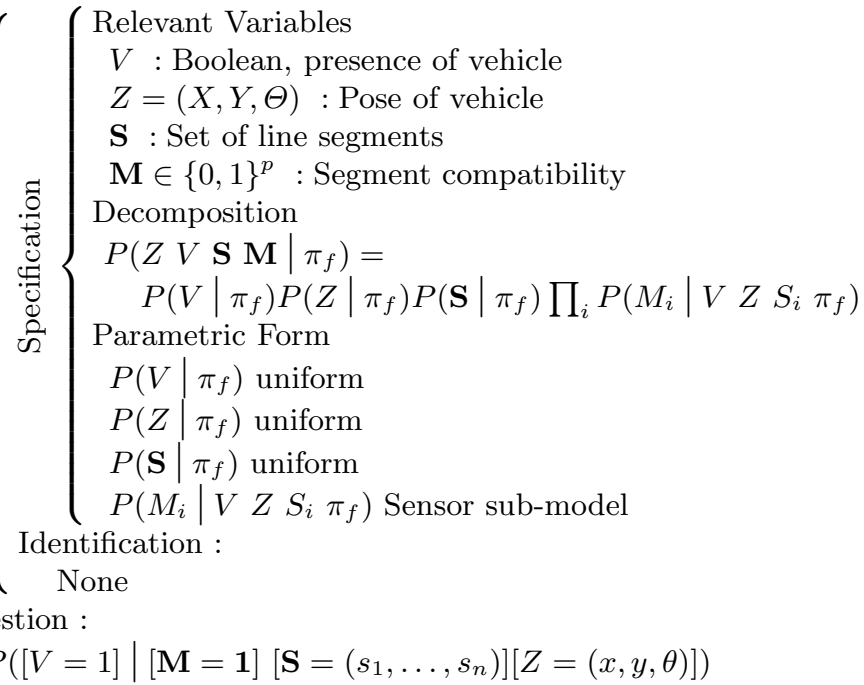 \\
\hline
\end{tabular}

Fig. 2: Detection of vehicles bayesian program

$\left.\left.\left(s_{1}, s_{2}, \ldots, s_{n}\right)\right][Z=(x, y, \theta)] \pi_{f}\right)$, gives the possible vehicles hypotheses. For ease of calculation, the logarithm is applied. The sub-model used in the

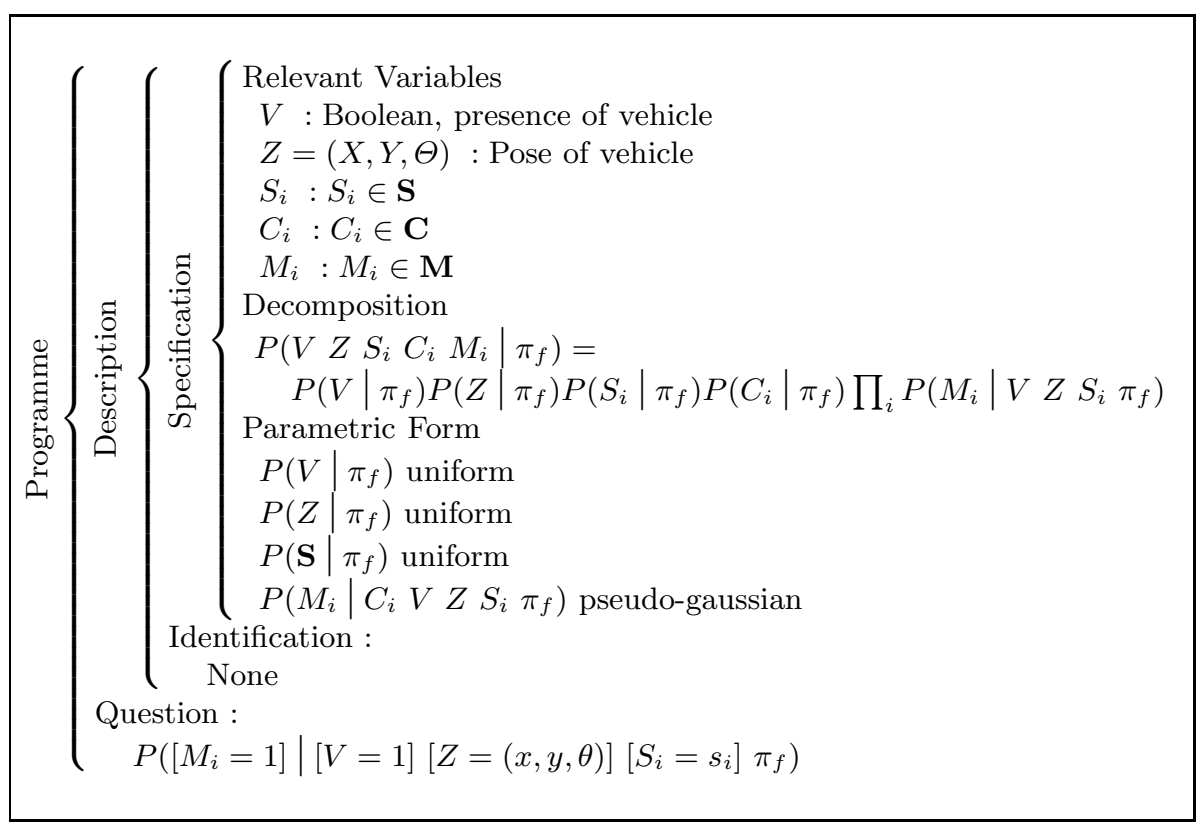

Fig. 3: Sensor Sub-Model bayesian program 
calculation of $P\left(M_{i} \mid V Z S_{i} \pi_{f}\right)$ is described in fig. 3. The question of the sub-model can be further resolved:

$$
\begin{aligned}
& \left.P\left(\left[M_{i}=1\right]|| V=1\right] Z S_{i} \pi_{f}\right) \\
= & \sum_{C_{i}} P\left(C_{i} \mid \pi_{f}\right) P\left(\left[M_{i}=1\right] \mid C_{i}[V=1] Z S_{i} \pi_{f}\right)
\end{aligned}
$$

The remaining problems lies in expressing $P\left(C_{i} \mid \pi_{f}\right)$, the probability that a segment corresponds to the length or width of a vehicle, and $P\left(\left[M_{i}=\right.\right.$ 1] $\left.\mid C_{i}[V=1][Z=(x, y, \theta)]\left[S_{i}=s_{i}\right] \pi_{f}\right)$, the probability that a segment and its given association to the sides of the vehicles, corresponds to a vehicle at pose $(x, y, \theta)$. For $P\left(C_{i} \mid \pi_{f}\right)$, a simple model is given by:

- $P\left(\left[C_{i}=1\right] \mid \pi_{f}\right)=\frac{L}{l+L}$

- $P\left(\left[C_{i}=2\right] \mid \pi_{f}\right)=\frac{l}{l+L}$

Given the length $\mathrm{L}$ and the width 1 of the assumed vehicle size, we consider the prior probability that the segment belongs to either the length or width is based on the simple ratio of the side in question against the sum of the two sides.

$P\left(\left[M_{i}=1\right] \mid C_{i}[V=1][Z=(x, y, \theta)]\left[S_{i}=s_{i}\right] \pi_{f}\right)$ is expressed by a pseudo-gaussian function $G\left(S_{c}, s_{i}\right)$. Where $S_{c}$ represents the segment of a side of the hypothesized vehicle position $(x, y, \theta)$. The more $s_{i}$ is further or have a different orientation with respect to $S_{c}$, the smaller the value of $G\left(S_{c}, s_{i}\right)$. In practice, as most of the values in the histogram are negligable if the histogram is to be filtered by a threshold, it is sufficient to go through the list of segments and filling in the histogram values only for the possible vehicle poses $(x, y, \theta)$ that are compatible with the line segments. The vehicle poses can be easily calculated with simple geometry.

\subsection{Edge Filtering}

To calculate how well the sides conform to a vehicle hypothesis configuration, the set of relevant segments around the contours of the hypothesized vehicles have to be selected. The filtered edges are to provide data for the calculation of the vehicle support (section 3.3).

Two bounding rectangles are calculated from the vehicle hypothesis configuration with one rectangle a ratio smaller than the original vehicle size and the other a ratio bigger as illustrated in figure 4 . The two bounding rectangles will then be oriented and positioned in the same manner as the hypothesized vehicle configuration.

The algorithm begins with the segment that contains the end point nearest to the origin (where Sick is). Starting from this segment, the algorithm starts to grow outward by searching for any segments where any of its endpoints lies sufficiently close to one of the endpoints in the original segment. This continues till a sufficiently close segment cannot be found. 


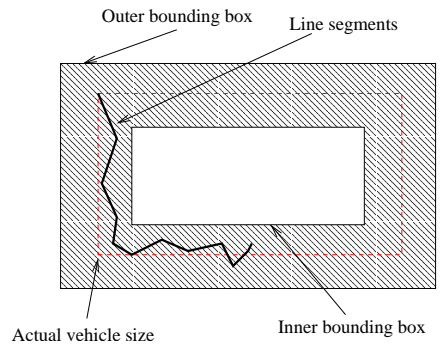

Fig. 4: Edge filtering with 2 bounding boxes. Shaded area indicates valid area

\subsection{Vehicle Support Calculation}

In adopting a conservative approach, both length and width of the vehicle must be adequately observed. Cases where either its length or width is verified are rejected as it introduces ambiguities and potentially false positives. A metric, the vehicle support, based on the sum of the magnitude of cross products can be used to enforce our conservative approach.

The calculation of vehicle support is given by eqn. 1 . Under the ideal case where there are only two segments perfectly aligned to the edges of the vehicle, the result is a multiplication of the length and width of the vehicle. The equation for calculating the support is given by:

$$
\text { support }=\forall i, j \sum_{i \neq j}\left|S_{i} \times S_{j}\right|
$$

Intuitively, if a large portion of the length and a small portion of the width is detected, the support will give a low value and vice versa. Hence it enforces the verification of both the length and the width of the hypothesized vehicle before classifying it as a positive vehicle hypothesis. The enforcement of such rules are all conveniently embodied in a single equation (eqn 1).

\section{Map Construction}

The SLAM algorithm is independent of the construction of the vehicle hypotheses. Hence, a variety of SLAM methods can be used. If multiple hypotheses SLAM algorithms are used, the different hypotheses might come up with conflicting hypotheses caused by association of landmarks. Such conflicting associations of landmarks must be resolved in order to obtain a final consistent map. In such cases, a single observation might be associated with different landmarks across the various SLAM hypotheses. The most likely group of hypotheses with the same data association for a given observation can be combined together to obtain the landmark to be represented in the 
final map while the rest of the hypotheses are ignored. A detailed description and example based on FastSLAM [5] [6], which is used in this paper, is presented in [9].

The bayesian programs used previously to construct the vehicle pose hypotheses gives a set of possible vehicle poses by taking each line segment extracted from the laser scan independently. It does not consider the previously made vehicle pose hypotheses. The disadvantage of the vehicle pose hypotheses construction and conservative gating approach is its inability to handle occlusion and false positives are obtained as a result. For example in fig. 5, a wrong hypothesis is constructed due to occlusion. A corner of a building is mistook as a vehicle pose hypothesis. By making more observations as CyCab navigates around to obtain a more comprehensive picture of the environment and taking into account previous hypotheses, wrongly made vehicle hypotheses can be removed.

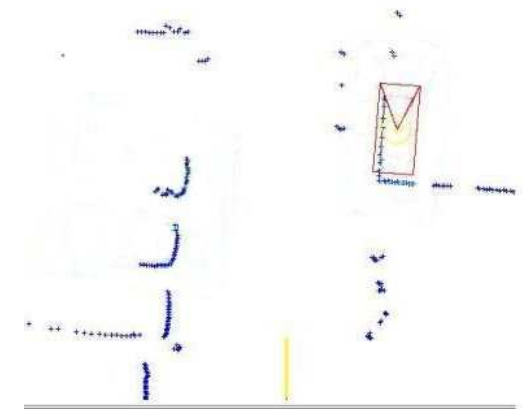

Fig. 5: An example of a wrong hypothesis due to occlusion

All hope is not lost for vehicle hypotheses that failed gating, often due to occlusion. Hypotheses that failed gating can be checked against previously accepted hypotheses. A current hypothesis that is very similar in position and angle with a previously accepted hypotheses can be taken to be a positive hypothesis by virtue of being previously accepted.

\subsection{Considering Previous Hypotheses}

The two main criteria for measuring similarity of a current hypothesis and a previously accepted hypothesis are their position and angle. It will be convenient to obtain a similarity measurement function that returns a bounded value of between 0 and 1 . The measurement of difference in position is given by function $f$ :

$$
f=\frac{2 \times \operatorname{Area}(P \bigcap Q)}{\operatorname{Area}(P)+\operatorname{Area}(Q)}
$$


Where $P$ and $Q$ are both the geometry of the two vehicle hypotheses. A perfect fit of $P$ and $Q$ gives a value of 1 and a non overlapping $P$ and $Q$ gives a value of 0 . Similarly, a measurement of difference in angle, $g$ :

$$
g=1-|\sin \theta|
$$

The two hypotheses are considered similar as long as $\min (f, g)>$ threshold.

\section{Experimental Results}

\subsection{Vehicle Detection}

After stage 1 (without gating), the vehicle hypothesis to the left in figure 6 is accepted even when only either the length of the vehicle is detected. But in fact, that detected side corresponds to a wall. This example demonstrates the weakness of using a laser scanner as ambiguous situations renders the system incapable of inferring correctly if it is vehicle. Additional information from a camera would be more useful. Such examples are the main motivation for a gating approach.

Stage 2 (gating) rejects the wrong hypothesis as represented by its inner and outer bounding boxes in fig. 7. Due to the conservative approach in validating hypotheses in stage 2 , some potential hypotheses are inevitably eliminated in the process (eliminated vehicle hypothesis to the right in fig. 7).

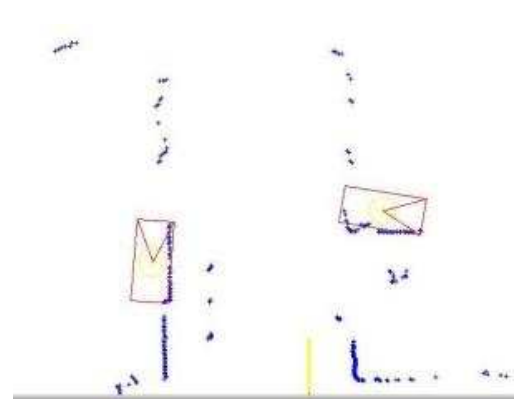

Fig. 6. Accepted vehicle hypothesis after stage 1 of vehicle detection

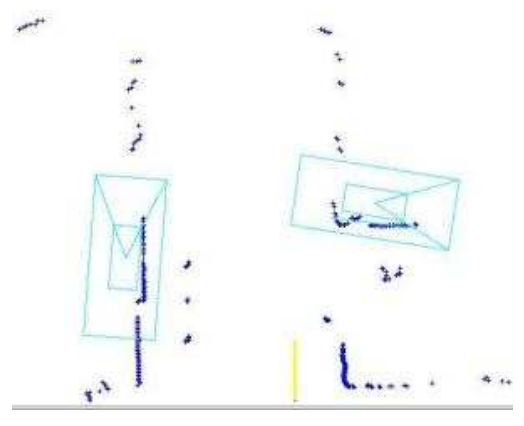

Fig. 7. The same configuration as in figure 6 but with rejected vehicle hypothesis in their inner and outer bounding boxes after stage 2 of vehicle detection

Vehicles parked in the car park are often spaced out sufficiently enough to be able to view its sides from the point of view of the laser scan. However, in the less likely event of being spaced very close together such that the relevant 
laser impact points cannot be obtained, the relevant hypotheses cannot be obtained. This is one of the limitations of using only a laser scanner. A fusion of data with a camera will be desirable.

\subsection{Map Construction}

Tests were conducted within the context of the car park in INRIA RhôneAlpes. The current implementation is a naive and unoptimized version of FastSLAM that executes with a frequency of between $4-6 \mathrm{~Hz}$ on a pentium 4 .

Figure 8 presents the multiple hypotheses of the position of CyCab and the other vehicles in the environment. The various CyCab hypotheses are followed by a curve indicating its mean path taken and the various landmark hypotheses.

Figures 9 and 10 illustrates final constructed map. It is basically a combined map of all the various hypotheses obtained from FastSLAM. In addition, the figure is overlaid with the laser impact points from the first laser scan and the laser scan at CyCab's current position.

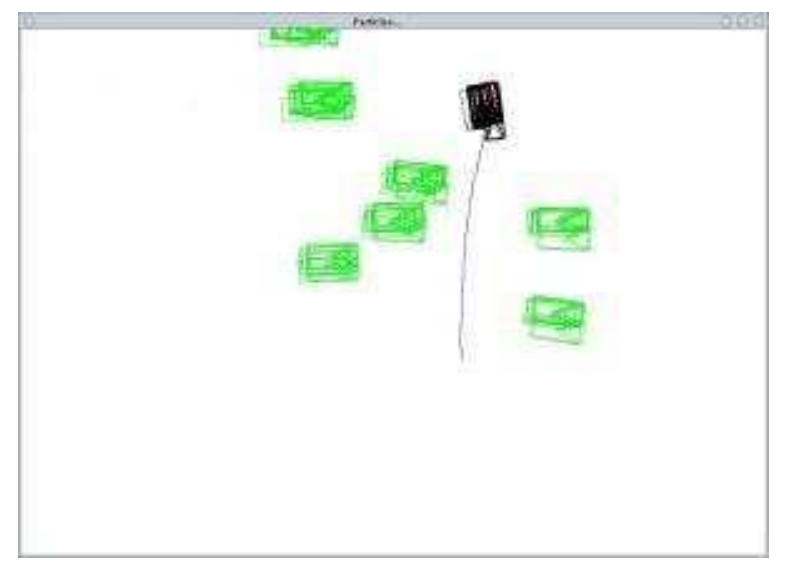

Fig. 8: The various CyCab and landmark hypotheses

\section{Conclusion and Further Work}

In this paper, the extraction of hypothesized vehicle poses from laser scan data only is presented. Due to limitations in the laser scan data, gating is required to remove the false positives. However the conservative vehicle hypotheses construction approach is at the cost of eliminating potential vehicle hypotheses. Vehicle hypotheses construction can be ameliorated by taking into account previously accepted vehicle hypotheses. 


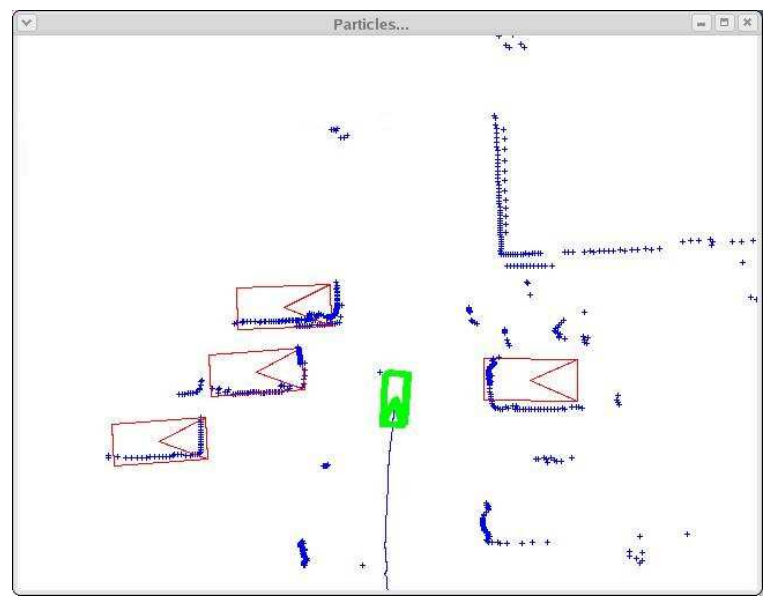

Fig. 9: Final map obtained by fusion of different hypotheses

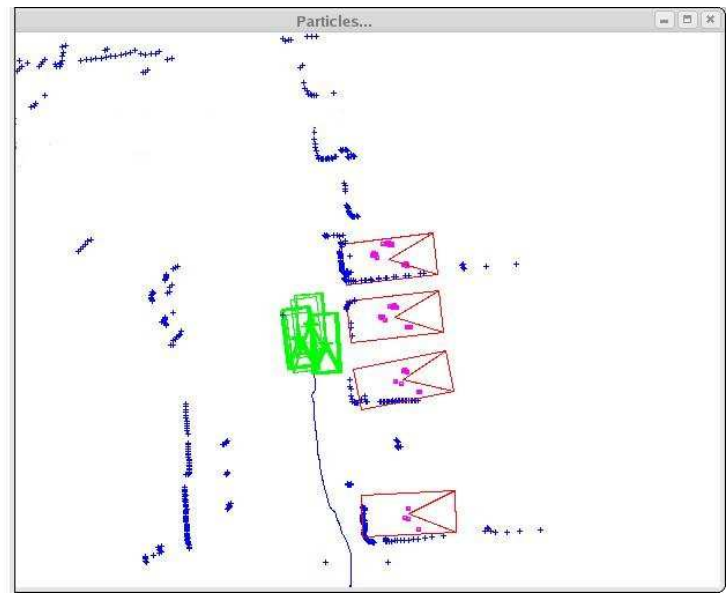

Fig. 10: Another mapping example

A more reliable method of vehicle hypotheses construction will be explored by fusing laser scan information with information from a camera. The current implementation assumes a static environment where landmarks do not move. Extensions will be made to handle moving objects.

\section{Acknowledgements}

This work is made possible by a study grant from the French Embassy of Singapore. 


\section{References}

1. C.Pradalier, J.Hermosillo, C.Koike, C.Braillon, P.Bessiere, and C.Laugier. An autonomous car-like robot navigating safely among pedestrians. In International Conference in Robotics and Automation, 2004.

2. O. Lebeltel. Programmation Bayienne des Robots. PhD thesis, Institut National Polytechnique de Grenoble, France, October 1999.

3. Y. Liu, R. Emery, D. Chakrabarti, W. Burgard, and S. Thrun. Using EM to learn 3D models with mobile robots. In Proceedings of the International Conference on Machine Learning (ICML), 2001.

4. C. Martin and S. Thrun. Online acquisition of compact volumetric maps with mobile robots. In IEEE International Conference on Robotics and Automation (ICRA), Washington, DC, 2002. ICRA.

5. M. Montemerlo, S. Thrun, D. Koller, and B. Wegbreit. FastSLAM: A factored solution to the simultaneous localization and mapping problem. In Proceedings of the AAAI National Conference on Artificial Intelligence, Edmonton, Canada, 2002. AAAI.

6. M. Montemerlo, S. Thrun, D. Koller, and B. Wegbreit. FastSLAM 2.0: An improved particle filtering algorithm for simultaneous localization and mapping that provably converges. In Proceedings of the Sixteenth International Joint Conference on Artificial Intelligence (IJCAI), Acapulco, Mexico, 2003. IJCAI.

7. C. Pradalier and F. Colas. Expressing bayesian fusion as a product of distributions: Applications in robotics. In IEEE/RSJ Int. Conf. on Intelligent Robots and Systems, October 2003.

8. Chatila R. and Laumond J.-P. Position referencing and consistent world modeling for mobile robots. In IEEE International Conference on Robotics and Automation, pages $138-145,1985$.

9. C. Tay, C. Pradalier, and C. Laugier. Vehicle detection and car park mapping using laser scanner. 2005. 